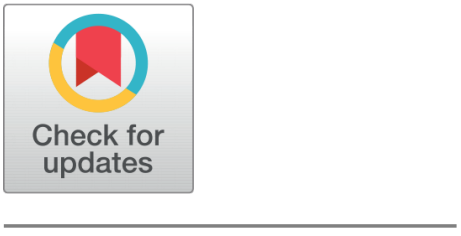

open ACCESS

Received: 01.01.2021

Accepted: 23.02.2021

Published: 01.03 .2021

Citation: Purohit S, Jain AK (2021) Leveraging IOT and AI for delivering tailor-made customer experiences in Indian petro retailing. Indian Journal of Science and Technology 14(7): 604-609. https://doi.org/ 10.17485/IJST/v14i7.3

* Corresponding author.

Tel: 9717094396

purohits13@gmail.com

Funding: None

Competing Interests: None

Copyright: () 2021 Purohit \& Jain. This is an open access article distributed under the terms of the Creative Commons Attribution License, which permits unrestricted use, distribution, and reproduction in any medium, provided the original author and source are credited.

Published By Indian Society for Education and Environment (iSee)

ISSN

Print: 0974-6846

Electronic: 0974-5645

\section{Leveraging IOT and Al for delivering tailor-made customer experiences in Indian petro retailing}

\author{
Santanu Purohit ${ }^{1 *}$, Arvind Kumar Jain ${ }^{1}$ \\ 1 School of Business, University of Petroleum \& Energy Studies, Dehradun, India. \\ Tel.: 9717094396
}

\section{Abstract}

Objectives: To explore the applicability and impact of deploying an IOT \& Al solution (CCTV-based solution) on customer experience at a fuel retail outlet. Method: CCTV-based IOT \& Al solution is used for capturing customer details, ensuring safety and service protocol implementation. A technologyled approach toward ensuring service delivery, enhanced customer experience and their satisfaction. The experiment was conducted at one of the fuel stations in the city of Mumbai and studied for the period of Aug- Oct 2020. The analysis is carried out on the data gathered during the period. Findings: Our research has shown the utility of a CCTV-based solution for identification, capturing \& accessing customer movement \& behavior on the fuel station forecourt. It is also found effective for capturing safe practices which intern gives a sense of safety thus leading to customer satisfaction. Thus, research showed that IOT implementation can yield significant benefits towards customer identification, increase in operational efficiency and customer acquisition, retention \& satisfaction thus leading to enhanced customer experience and increased sales. Novelty : The study is the first of its kind towards the application of CCTV-based solutions on customer experience in Indian Petro Retailing.

Keywords: IOT; Indian fuel retail; IOT in oil \& gas; new technology adoption; customer experience; customer identification

\section{Introduction}

Consumer Buying Behavior, which involves the decision processes and acts of people involved in buying and using products, has gained importance in the last few decades. Indian fuel retail market has experienced the evolution of the concept of fuel retail from a commodity to a service-based product over the last two decades. The earliest models of Fuel Retail involved simple fuel sales. However, over a period of time, and due to competitive market scenario, fuel retail companies started providing various additional services like Free Air for Vehicle, Tyres, Car Wash, Quick Car Check-up and Service, Convenience Stores, Quick Serve Restaurants, Kids Zone, Clean toilets. Recently, there have been a lot of direct marketing initiatives to attract and retain the customers viz; Loyalty Programs, Cash Back, Free Gifts, Promotions \& Schemes ${ }^{(1)}$. Customer buying 
behavior has also evolved with the concept of availing value-added services leading to a service-based industry. Societal technological advancement has led to this change ${ }^{(2)}$. As a result, customer desire has also enhanced \& evolved towards getting value-added services under one umbrella, forcing oil marketing companies (OMCs) to develop large format fuel stations which offer many additional services. Consumer experience has become more sophisticated as consumer buying behavior and expectations evolved over time ${ }^{(3)}$.

While the retail sector (excluding petro-retail), has witnessed huge digitization in operations, customer experience, understanding of customer needs and building loyalty, the petro-retailing sector is yet to catch up to comparable levels. In India, digitization has become a key facet of almost every sector of retailing, impacting the buying behavior of consumers at large. This has forced OMCs to accelerate their technology adoption. OMCs have been trying to introduce value-added services with limited technological advancement ${ }^{(4)}$.

Rapid technological advancements, as well as increasing exposure to digitization, have led to a more evolved modern customer. Accordingly, customer habit, expectation \& experience, in general, have undergone a change and still evolving. The retail sector has witnessed a remarkable shift in customer buying behavior due to technological enhancement ${ }^{(5)}$. There is overlap among customers in retail and petro-retail as well. Customer behavior towards petro-retailing is also evolving. Adoption of technologies like AI, big data, cloud, mobile technology, etc. in the upstream part is quite mature ${ }^{(6)}$. However, in the Indian context, the same is not very significant on the downstream side, particularly in the areas of customer identification, recognition, meeting their expectations of product/service mix, etc. along with integrated, seamless, and touch-less transactions. While General retail has made rapid advances in technology implementation and thus led to enhanced customer expectations, petroretailing is yet to catch up ${ }^{(4)}$.

Petro retailing in India was a commodity business under highly regularized conditions. However, with the passage of time, it has become service-based and additional services other than fueling which are need of the hours, specifically in an urban market. With the exposure of the latest technology, increasing engagement and usage of technology has led to a shift in customer buying behavior $^{(7)}$.

Technology has also influenced the nature of services themselves, how they are delivered, and the practice of service innovation and service management. The retail industry has undergone a huge change globally in terms of technology adoption. While this change has been very pronounced and visible in general retail, fuel retail has also had its fair share of technology innovations. This scenario holds true in the Indian context also. Indian retail has undergone a sea change while the Indian Fuel retail market has evolved from commoditized sales to service-based product offerings over the last few decades. However, technology adoption including IoT continues to be at a marginal level ${ }^{(8)}$. It has also been observed that there is limited availability of solid frameworks \& models that can address the problems in Service and there are several barriers to the implementation of IoT solutions from both technological and managerial perspectives ${ }^{(9)}$.

In the current era, customers have exposure to social media and technology adaptation in the retail sector. This has enhanced the desire for technological-based advancement in products and services ${ }^{(10)}$. With the shift in customers buying behavior in petro retailing, customer expectation has also evolved. Usage of RFID technology towards identification, recognition, seamless transaction are important touchpoints in the customer's buying journey ${ }^{(11)}$. Identification of customers before fueling is very much important and customer expectations get upgraded for better service desire and satisfaction. Hence adaptation of suitable technology for identifying customers, ensuring service delivery standards and safety \& security at the fuel station plays an important role towards customers' enhanced experience thus satisfaction ${ }^{(12)}$. IOT-based fuel station alert systems have also been tried to determine the probable design for generating alerts in case of theft of fuel and introduction of a security matrix for appropriate communications ${ }^{(13)}$.

\section{Methodology}

In the current scenario of petro retailing in India, service delivery is being monitored, ensured predominantly through manual processes where human interventions are required periodically. Though OMCs have provided an automation system at the fuel outlets, this is mainly being used for stock \& transaction monitoring and tracking. Presently, usages of the automation system towards customer service delivery are negligible. Some critical activities towards service delivery to customers like Customer Identification, Ensuring service standard at a fuel station, Ensuring safety practices, guiding the customer to the vacant island, are currently being done manually at fuel outlets.

With vast development in technology using computer vision, image processing, artificial intelligence and machine learning, usage of such technology can help to develop a solution to analyze videos recorded at the fuel stations and derive these insights from them which will help service provider companies to improve on (i) Customer identification for enabling personalization, asset utilization, (ii) customer experience at the fuel station along with the positive impact of (a) Manpower availability, (b) Service standard improvement, (c) Traffic management, and (d) Fuel station operations \& safety. 
Knowing the customers and their experience at fuel stations will help service providers to further put efforts in winning new customers and retaining the existing customers. The majority of fuel stations in India have been provided CCTV cameras for monitoring and safety \& security purposes. In our study, the footage of CCTV cameras has been analyzed using machine learning and artificial intelligence to understand customer visiting patterns and knowing about their experience is to leverage the CCTV footage at fuel stations. In the experiment, Edge computing technology was also used which broadly means all the computing happening at the edge of the network without depending on back end infrastructure and connectivity medium for transmitting the data.

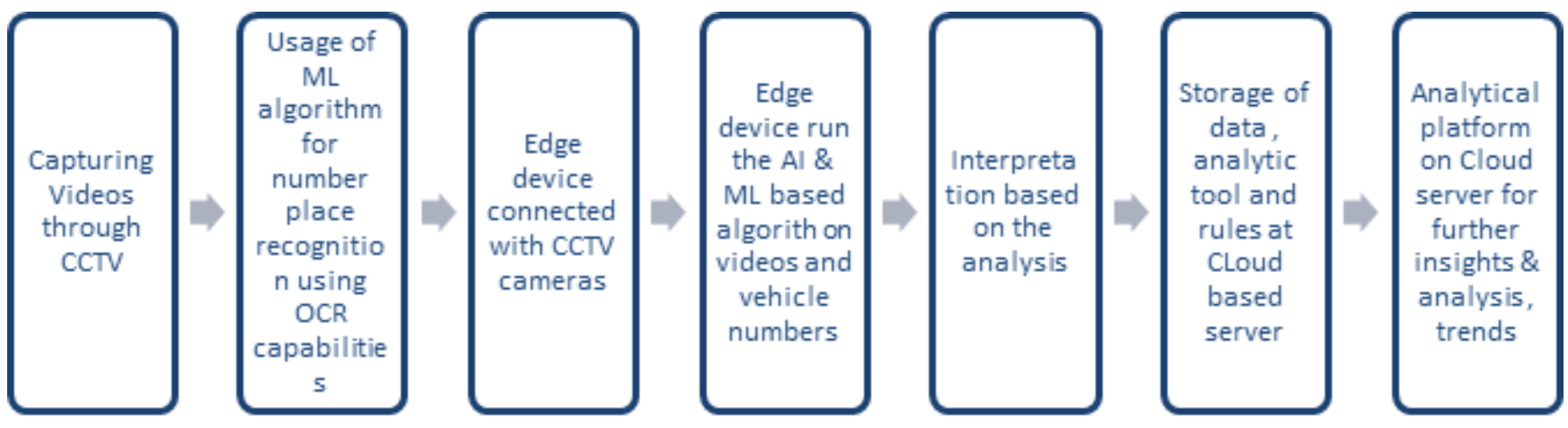

In our study we have focused to capture (i) queue patterns at the fuel station, (ii) reading \& recognizing the vehicle number plate to identify the customer by means of vehicle number (iii) identifying safe operation at the retail outlet. Tank lorry carrying fuel gets decanted in the underground tank at the fuel station and based on the standard operating practice of fuel station, it is one of the critical activity so far safety is a concern. Hence, in our study, we have tried to identify the deviation in this operation.

Also, a survey was conducted on 102 customers at the retail outlet pre and post-implementation of the solution to assess the improvement and impact on customer behavior, satisfaction and confidence on fuel station.

\section{Key Components of the Solution}

The key component used in our study are:

- CCTV (IOT device)

- Edge Computing device with AI \& ML algorithms

- Cloud base backend analytical platform.

The solution is based on the footage available from CCTV and running analysis on the footage on real-time basis. Machine learning algorithms were developed based on the objectives and the model was trained extensively for the specific scenarios. The algorithm was developed on open source tools and has Optical Character Recognition (OCR) capabilities to read the number plates of vehicles. With the help of OCR capabilities image of the vehicle number plate got converted to characters i.e Alphabets \& numbers. Edge computing system deployed at each of the fuel stations which is capable to manage all the data coming from all the CCTVs deployed at the fuel station for the purpose. The edge computing system consisting of one or more single-board computers with enough graphic processing power to collect all the CCTV data and run the AI \& ML codes on them to derive the necessary insights. Artificial intelligence algorithms were developed for autonomous decision-making, interpreting and notifying concerned officials regarding points of interest without any manual intervention. At the back end, a Cloud based analytics platform was provided that further derived insights from the data received from the Edge devices.

\section{Results \& Discussion}

The work is first of its kind of primary work while previous works were based on the secondary work. This primary work with the technology-based solution was observed and experienced for three months and learning \& evaluation is carried out based on the data available from the experiment. Real-time data were analyzed w.r.t the objectives and the performance observed is depicted in Table 1 . The solution showed $100 \%$ results on traffic count and ensuring safety parameters. 
Table 1. Performance of objectives

\begin{tabular}{lll}
\hline Sr no & Objectives & Performance against expectation \\
\hline 1 & Traffic count & $100 \%$ \\
$1 \mathrm{a}$ & Queue Management & $95 \%$ \\
2 & Autonomous number plate recognition & $92 \%$ \\
3 & Ensuring Safety & $100 \%$ \\
\hline
\end{tabular}

In the case of Autonomous number plate recognition (ANPR) using OCR capability of Edge computing device, the customer was identified with the help of vehicle number recognized by the system and customer identities like name, mobile number, etc. which were pre-defined in the backend cloud server as a part of enrollment activity. The solution has the capability to enroll new customers as well during their first visit to the fuel station. Data gathered over three months were analyzed for (i) Segmentation of Customers at fuel station i.e. type of vehicle like 2 wheeler, 4 wheeler, etc. (ii) Accessing footfall frequency of customers i.e. frequency in visiting the fuel station, (iii) Enrolling regular customers in loyalty programs based on their frequency of visit \& fuel consumption pattern, (iv) Visibility of Regular customers, (v) Enhanced Customer experience for customers with real-time information of their presence at a fuel station, (vi) Targeted Marketing, (vii) Automated Greeting of Customers using existing automation system at the fuel station. The observation has further been analyzed on customers visited at the fuel stations during the observation period and their contribution to the revenue of the fuel station.

The survey was conducted on 102 customers who visited the fuel station. It was observed that the majority of the customers are male (92\%) and are in the age bracket from $30 \mathrm{yrs}$ to $40 \mathrm{yrs}$. Analysis of primary data shows that majority of revenue is contributed by Cars $(84.30 \%)$ at the fuel station. A survey on the customer feedback and demography analysis is depicted in Tables 2 and 3 . The feedback on their experience shows that $10.80 \%$ of customer's experienced satisfactorily (Table 4 ) which indicates that the solution has a positive impact on customer experience and satisfaction.

The experiment with respect to the safety practices shows that safety alarms generated once defined safe practices have not adhered to. The solution also provided information on the basic service standards i.e adherence of service protocol, attire and behavior of service delivery man, service at the overall fuel station and air filling points.

Table 2. 4 Wheeler vehicle population \& contribution to revenue

\begin{tabular}{lll}
\hline Type of Vehicle & Population & Contribution to revenue \\
\hline Bus & $4.80 \%$ & $4.70 \%$ \\
Truck & $10.30 \%$ & $11 \%$ \\
Car & $84.90 \%$ & $84.30 \%$ \\
\hline
\end{tabular}

$\left({ }^{*}\right.$ source - Primary data $)$

Table 3. Demographic analysis of customers (base 102 Customer)

\begin{tabular}{llll}
\hline Gender & Population & Age & Population \\
\hline Men & $92.00 \%$ & $20-30$ year & $4.20 \%$ \\
Female & $8.00 \%$ & $30-40$ year & $89 \%$ \\
& & $40-50$ year & $6.80 \%$ \\
\hline
\end{tabular}

$\left({ }^{*}\right.$ source - Primary data $)$

Table 4. Customer feedback (base 102 Customer)

\begin{tabular}{ll}
\hline Experience & Population \\
\hline Happy & $10.80 \%$ \\
Disappointed & $10.20 \%$ \\
Neutral & $89.00 \%$ \\
\hline
\end{tabular}

( ${ }^{*}$ source - Primary data) 


\subsection{Impact on fuel station}

Fuel station owner/operator accurately knows the vehicles/customers visiting the fuel station and can have targeted communication to select customers since frequency and value generated by customers are known to them now.

\subsection{Impact on customer}

The CCTV based IOT solution ensures the traffic and queue management at the fuel station thus provides a clear guideline for the customer for better fuelling experience. Also, ensuring the safety practices provides customers a sense of a safe \& secure environment at the fuel station.

\subsection{Impact on service providers (Company)}

The solution enabled service providers to capture customer details for every transaction and customer insight is available for the fuel station. Going forward, it can be available for the network if it is implemented across. The service providers have the visibility of customer buying behavior and have information to design a differentiated value-based offering for the customers. The solution helps the OMCs to monitor customers fueling pattern thus enabling them to develop an effective approach to build a lasting relationship. Also, OMCs can monitor the implementation and adherence of service protocol, safety practices at all of their fuel station. This will not only help to adherence to the protocols, in long run, but it will also enhance the overall service quality delivery and brand image of the company.

\section{Conclusion}

The CCTV-based IOT system has truly emerged as a differentiator and will definitely pave the way for the service provider to bag the crown of the market leader. Haphazard Traffic and queue during peak hours at the fuel station create an unsatisfactory experience for the customers. With this technology implementation, the customers are guided to the formation of lanes and guided to the available free Island thus leading a pleasant experience for them. Usage of ANPR and capturing customer details enable service providers to design customized communication, a promotional program based on their buying pattern \& behavior. This will also enable OMCs to work on various business models of incentivizing their network, differential commission, rate and reward the dealer/ delivery man/ forecourt personnel based on the customer behavior and sales pattern in the longer run.

The CCTV based IOT solutions have a positive impact in the areas of (i) Enhanced Customer Experience where customer aims to have a smooth fueling experience without any hiccups at the fuel stations including personalized programs \& communication (ii) Increase in Operation Efficiency by reducing the fueling cycle time, proper traffic management, adhering safety practices (iii) enhanced brand building including new customer acquisition and retention and (v) leading to additional revenue to the service provider. Thus, rapid technological advancements, as well as increasing exposure to digitization, have led to increasing adoption of digital technologies, including IOT \& AI-based solutions. In order to offer a seamless experience to the customer when they visit fuel stations, there is a dependency on infrastructure at the outlet and the technical solution functionalities available at the fuel station. OMCs want to provide a robust platform of vehicle/customer identification so that the transactions can be tagged to customers and the process of receipt generation can be simplified. CCTV-based IOT solution is one step towards providing a better seamless buying experience at the fuel station. The solution provides a better experience to the customers and at the same time, it has a positive impact on all stakeholders such as fuel stations, customers and service providers i.e. OMCs.

\section{Future Scope}

In this article, we have tried to articulate how CCTV-based IOT technology can be helpful in the fuel retailing business as well from a service provider \& customer perspective. The study has been conducted at one fuel station in one city only. Viewing the encouraging outcome of the study, there is a scope to adapt and scale up the implementation of the technology by OMCs across for further enhancement in customer buying experience and satisfaction in fueling journey. Also, there is scope for an increase in the number of parameters for further study. 


\section{References}

1) Srinivasan T. A study on consumer preferences of petroleum retail outlets. IOSR Journal of Business and Management (IOSR-JBM). 2015;17(2):35-40. Available from: https://doi.org/10.9790/487X-17223540.

2) Yadav S, Sakariya S, Thaker M. Petro Retail Mix Elements: A Study Of Indian Market. Journal Of International Business And Economics;12(2):34-45.

3) Kumari RK, Devi NY. A study on consumer behaviour towards retail petrol outlet services in Coimbatore city. International Journal of Applied Research. 2016;2(2):670-673.

4) Purohit S, Jain AK. Changing Face of Digitization in Indian Petro- Retail: From Enterprise to Customer. International Journal of Multidisciplinary Education Research. 2020;9(6):121-129.

5) Farah MF, Ramadan ZB, Harb DH. The examination of virtual reality at the intersection of consumer experience, shopping journey and physical retailing. Journal of Retailing and Consumer Services. 2019;48:136-143. Available from: https://dx.doi.org/10.1016/j.jretconser.2019.02.016.

6) Digital Technology trends in Oil and Gas Industry by Dean Baker, RISC (UK), GEO ExPro. 2019.

7) Purohit S, Jain AK. Evolution of Fuel Retail In India Vis -a- vis India Customer : Shift in Consumer Behaviour. International Journal of Management ( IJM). 2020;11(8):199-207. Available from: https://doi.org/10.34218/IJM.11.8.2020.019.

8) Purohit S, Jain AK. Technological transition from analog to internet of things ( IoT ) on Indian petro-retail's customer service. Indian Journal of Science and Technology. 2020;13(42):4364-4368. Available from: https://doi.org/10.17485/IJST/v13i42.1964.

9) Mohamed HBD, Elkafi B, Zied. Internet of things and supply chain management: a literature review. International Journal of Production Research. 2019;57:4719-4742. Available from: https://www.tandfonline.com/doi/full/10.1080/00207543.2017.1402140.

10) Moran G, Muzellec L, Nolan E. Consumer Moments of Truth in the digital context: how 'search' and 'e-word of mouth' can fuel consumer decision making. Journal of Advertising Research. 2014;54. Available from: https://doi.org/10.2501/JAR-54-2-200-204.

11) Venkatesh J. RFID Technology: Improving Efficiencies and creating a better customer experience in retail Industry. International Journal of Management , IT \& Engineering. 2016;6(12):81-94.

12) Bigelow P. Chasing the data storm: Vehicle information can open opportunities in retail and safety- if drivers are willing to share it, Automotive News. .

13) kumar PN, Kumaresan P, Sundaresan YB. IoT based retail automation of fuel station and alert system. IOP Conference Series: Materials Science and Engineering. 2017;263. Available from: https://dx.doi.org/10.1088/1757-899x/263/4/042072. 